\title{
The Nightside Ionosphere: Ionospheric Convection during an Isolated Substorm on October 21, 1981
}

\author{
J. J. Moses ${ }^{1}$ and R. A. HeELIS ${ }^{2}$ \\ ${ }^{1}$ Astronomy Unit, Queen Mary and Westfield College, Univ. of London, London, U.K. \\ ${ }^{2}$ Space Sciences Center, University of Texas at Dallas, Richardson, Texas, U.S.A.
}

(Received September 18, 1995; Revised June 3, 1996; Accepted June 3 , 1996)

\begin{abstract}
Substorms can occur in serics such as during a geomagnetic storm or as isolated events. We have chosen an isolated substorm from the Dynamics Explorer-2 (DE-2) database and use the Expanding/ Contracting Polar Cap Model to study the substorm-related ionospheric electric fields and polar cap dynamics. The isolated event of October 21,1981 took place during several consecutive DE-2 passes which occur before the onset, just after the onset, during the substorm maximum, and during the recovery phase of the substorm. The polar cap expands during the growth and expansion phases and contracts little throughout the recovery phase. Tail reconnection, however, dominates the recovery phase. A total of $4 \times 10^{8}$ webers of open flux were reconnected during the recovery phase and removed from the polar cap.
\end{abstract}

\section{Introduction}

Over the past few years, a considerable effort has been made to describe the nightside ionospheric dynamics particularly during substorms (Lockwood et al., 1988; Moses et al., 1989, 1994). For this endeavor, it is quite useful to assume a rather simple model for the ionospheric convection which uses the fact that reconnection or the exchange of flux in the magnetosphere drives the ionospheric flows. Open magnetospheric flux tubes created at the dayside magnetopause by magnetic field merging are dragged across the polar cap thus driving antisunward polar cap flow. Conversely, reconnection in the magnetotail converts open flux back to closed on the nightside. The Expanding/Contracting Polar Cap (ECPC) Model can be used in concert with spacecraft data to provide insight into reconnection processes at work both on the dayside and nightside magnetosphere.

In this paper, we used the ECPC model and DE-2 satellite data to study an isolated substorm. The isolated substorm we chose to study occurred on Oct. 21,1981 . The event is covered by four consecutive DE-2 passes, one during the growth phase, one eight minutes past the onset, one just past the maximum of the substorm, and one later in the recovery phase. This fortuitous collection of passes allows us to observe the time history of the formation of the nightside gap and also the exchange of flux across the polar cap boundary.

\section{The Model}

Figure 1 gives a diagram of the basic features of the Expanding/Contracting Polar Cap (ECPC) model. The ionosphere is treated as a two-dimensional incompressible fluid. In this model, the polar cap boundary is represented by an adiaroic line which means that the polar cap boundary moves in response to the convection rather than ionospheric flows crossing the boundary. There are two possible gaps in the boundary through which flow can either enter or leave the polar cap. The dayside merging gap allows open flux to enter the polar cap and is the projection of the dayside merging line on the ionosphere. The nightside reconnection gap allows open flux to cross over to the closed field line region and maps to the tail reconnection $\mathrm{X}$-line. The adiaroic boundary connecting the dayside and nightside gaps will expand if the 


\section{Expanding/Contracting Polar Cap Model}

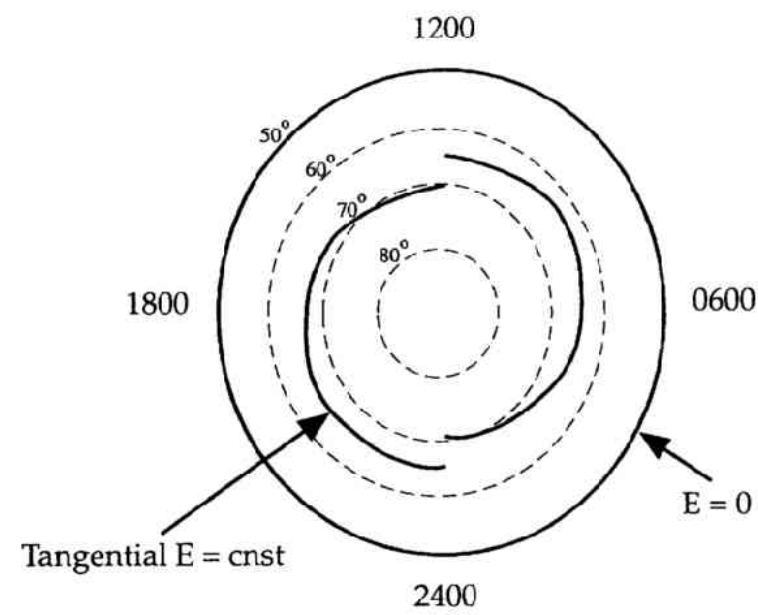

Fig. 1. Schematic of the ECPC model boundary conditions on a polar dial. For illustration, the dayside gap has been placed at noon and the nightside gap at midnight local time. The orientation of the dayside gap is correct for positive (negative) IMF $B_{y}$ in the northern (southern) hemisphere. The orientation of the nightside gap along a meridian is the most common. The potential drop across the gaps is fixed and evenly distributed along the polar cap (adiaroic) boundary. This gives uniform expansion/contraction of the polar cap boundary. On the outer shielding boundary here shown at $50^{\circ}$, the electric field goes to zero.

entry of open flux to the polar cap exceeds the loss and will contract if the loss of flux through the nightside gap exceeds the source. Potential drops are placed across the gaps to drive the ionospheric convection pattern. The adiaroic boundary is allowed to uniformly expand or contract which means the tangential electric field is constant along the boundary and the gap potential is uniformly distributed along it. The tangential electric field along the polar cap boundary is zero (i.e., an equipotential) only when the dayside and nightside gap voltage drops are equal. In this case, there is a balance between dayside and nightside reconnection rates; the net open flux in the tail lobes is constant; and, the polar cap boundary is stationary (i.e., $\boldsymbol{E} \times \boldsymbol{B}=0$ along the boundary). At lower latitudes, the model electric field and potential are forced to be zero corresponding to a perfect shielding layer. We solve for current continuity in the ionosphere (Eq. (1)).

$$
\nabla \cdot J=\nabla \cdot[\Sigma E]=0
$$

Input parameters for the model were obtained from the DE-2 satellite. The adiaroic line is drawn using the polar cap boundary locations obtained from the low altitude plasma instrument (LAPI) and information on the location of the dayside and nightside gaps. Proximity to the spacecraft track of the dayside merging gap is determined by the presence of magnetosheath or cusp precipitation on IMF $B_{y^{-}}$ directed flows detected by the ion drift meter/retarding potential analyzer (IDM/RPA). The nightside reconnection gap location depends on the presence of a band of strong flows with an eastward component embedded in the boundary plasma sheet precipitation. Examples of nightside gap signatures are further discussed below. The potential drops across the dayside and nightside gaps produce the electric fields in the polar cap and return flow regions. The gap potential drops are obtained from the integrated potential from vector electric field instrument (VEFI) measurements. After determining the location of the polar cap boundaries and whether a gap was present near the orbit track of the satellite, the adiaroic boundary 
is drawn as a semicircle as generally indicated by auroral images (Craven and Frank, 1985).

After running the model, the resulting convection pattern can be compared to the ion drifts from the IDM/RPA and the electric field component along the spacecraft track from VEFI. If necessary, the gap potentials can be readjusted and the model run again. More details on how potential patterns are obtained with the model are available in Moses et al. $(1987,1988,1989,1994)$ and Moses and Siscoe (1988).

Day 81294
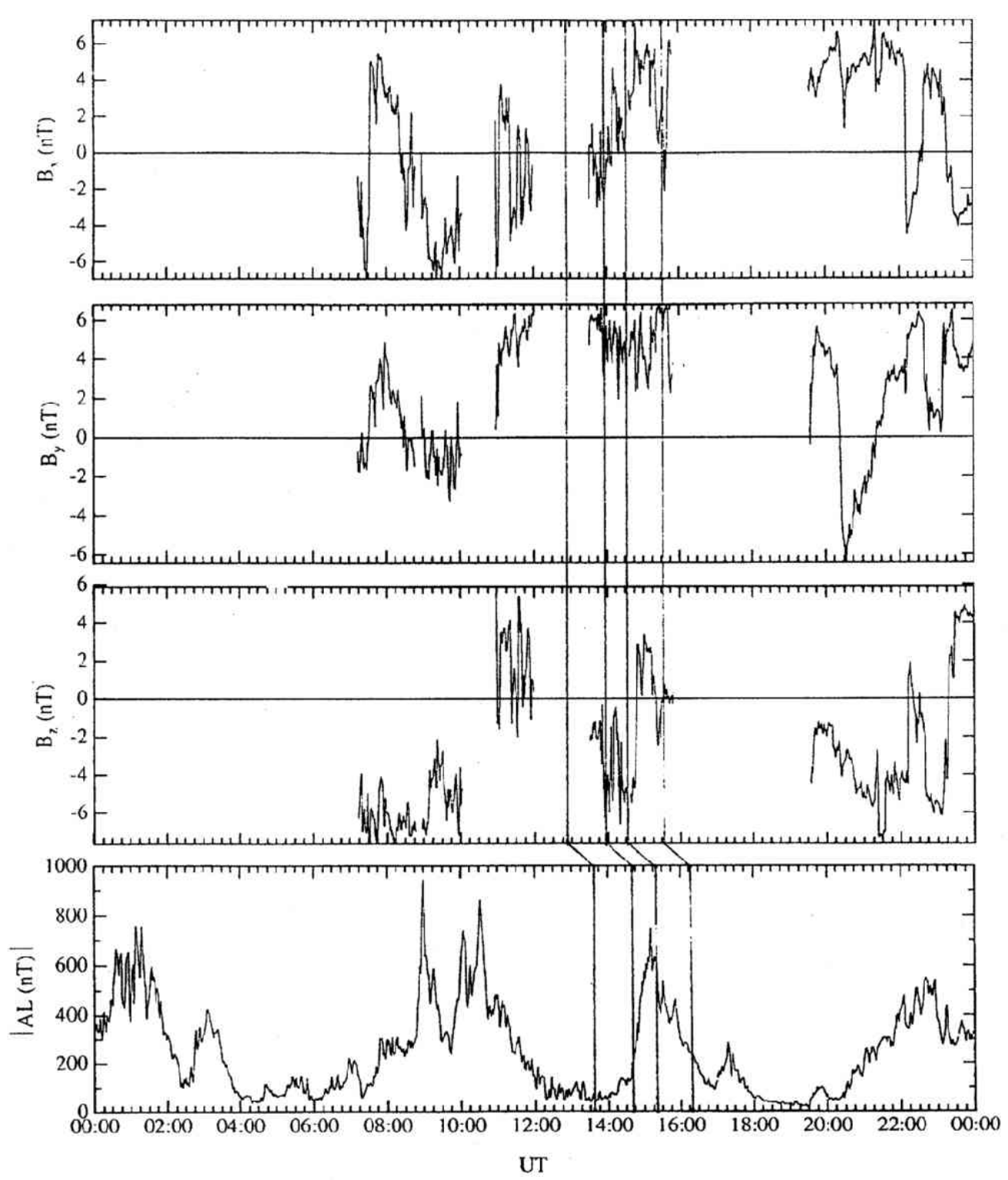

Fig. 2. ISEE-3 solar wind magnetic field and the magnitude of the $A L$ index for Oct. 21,1981 . The three panels from the top down display IMF $B_{x}, B_{y}$, and $B_{z}$. The times that the DE-2 satellite passes crossed the nightside polar cap boundary are marked with the vertical line. 


\section{Data}

We chose an isolated substorm for our study from a group of sustorms which fit the following criteria:

1. There must be no substorm onsets for 1 hour prior to the onset of our event.

2. The $A L$ index must be $>-100 \mathrm{nT}$ before any growth phase activity begins.

3. The event must completely recover $(A L>-100 \mathrm{nT})$ before the onset of the next substorm. The type of events singled out by the criteria above are rarely associated with large geomagnetic storms and often coincide with weak southward IMF or northward turnings of the IMF.

We define the substorm expansion and recovery phases by the rise and fall of the $A L$ index. The expansion phase is defined when the $|A L|$ index is increasing. Recovery is defined when $|A L|$ is decreasing. The growth phase cannot be readily defined from the $A L$ index. We identify the growth phase in this case by an expanding polar cap. Also, DE-1 auroral images are available at the time of this event giving us a more accurate determination of substorm onset.

Figure 2 shows the ISEE-3 solar wind magnetic field and the magnitude of the $A L$ index for Oct. 21 , 1981. The isolated substorm occurs at the very end of a geomagnetic storm and during a period of weak IMF $B_{z}$. The substorm or the recovery of the substorm isn't apparently triggered by a northward turning of the IMF. The times the DE-2 satellite crossed the nightside polar cap boundary are marked for each pass with the $\sim \mathbf{4 5}$ minute lag for the ISEE-3 data noted. The first DE-2 pass in the series crosses the nightside polar cap boundary at 13:39 UT. As one can see from Fig. 2, geomagnetic conditions are quiet. The DE1 images show a uniformly weak auroral oval for the first pass in this study consistent with the complete recovery from the previous event. According to the auroral images, the onset for the substorm occurred at 14:34 UT. The $A L$ index seems to lag the auroral images by $~ 7$ minutes. The second DE-2 pass occurs at 14:42 UT, eight minutes after the onset. Between the growth phase pass and the onset pass, the IMF turns southward and remains southward until 14:46 UT. DE-2 crosses the nightside polar cap boundary at 15:20 UT in the third pass. From the $A L$ index, the recovery phase has already begun about ten minutes before. The IMF has turned northward by 16:17 UT when the recovery phase has been in progress for over an hour for the fourth DE-2 pass in the study.

\section{Nightside Gap Signatures}

The signatures of the nightside gap and the polar cap boundary are quite distinctive from each other as seen in Fig. 3. Figure 3 displays the ion drift velocities from each DE- 2 pass in the study. Figure 3 a shows the ion drifts from the first pass. This is an example of a nightside gap with the usual meridional orientation. Between $72^{\circ}$ and $76^{\circ}$ invariant latitude in the evening local time sector lies the region of the boundary plasma sheet precipitation. Also, the flow has a significant eastward component within these latitudes. Ion drift velocities are significantly enhanced in this channel of flow over those in the polar cap. This signature of a nightside gap is the most common.

Figure $3 \mathrm{~b}$ pictures the ion drifts from the second pass in the series consistent with an expanding polar cap. The nightside polar cap boundary lies $\sim 68^{\circ}$ invariant latitude. Just equatorward of that boundary lies the convection reversal. Figure $3 \mathrm{~d}$ shows ion drifts from the fourth pass and an example of a latitudinal orientation of the nightside gap. The polar cap boundary lies at $\sim 75^{\circ}$ invariant latitude. The convection reversal lies around $10^{\circ}$ equatorward of the polar cap boundary. This is the signature of the latitudinal nightside gap. Flow exits the gap at the polar cap boundary and proceeds equatorwards on closed field lines. In the previous case (Fig. 3b), flow reverses close to the polar cap boundary consistent with the polar cap expanding with the flow. 
a.)

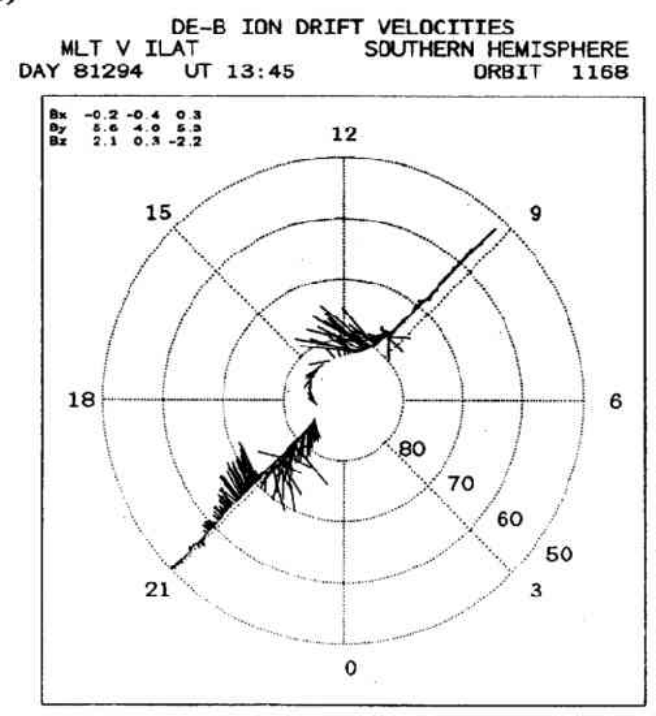

C.)

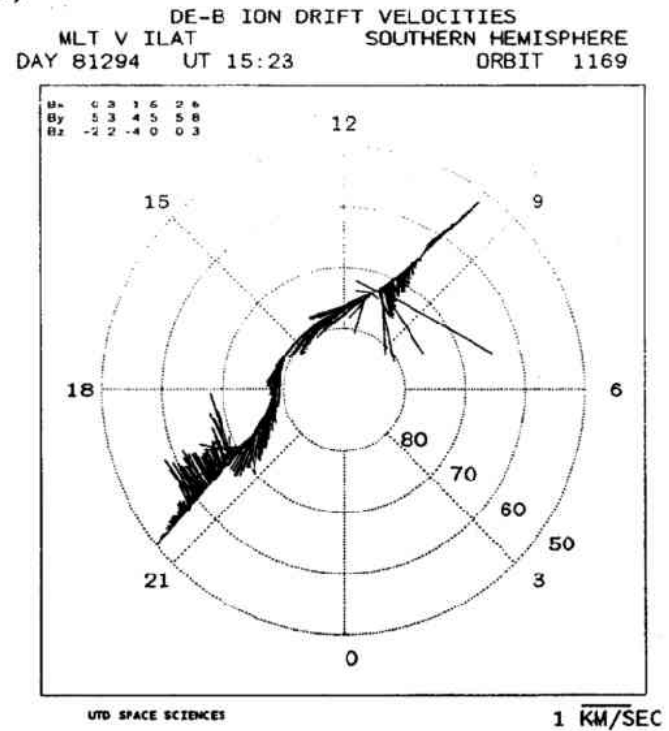

b.)

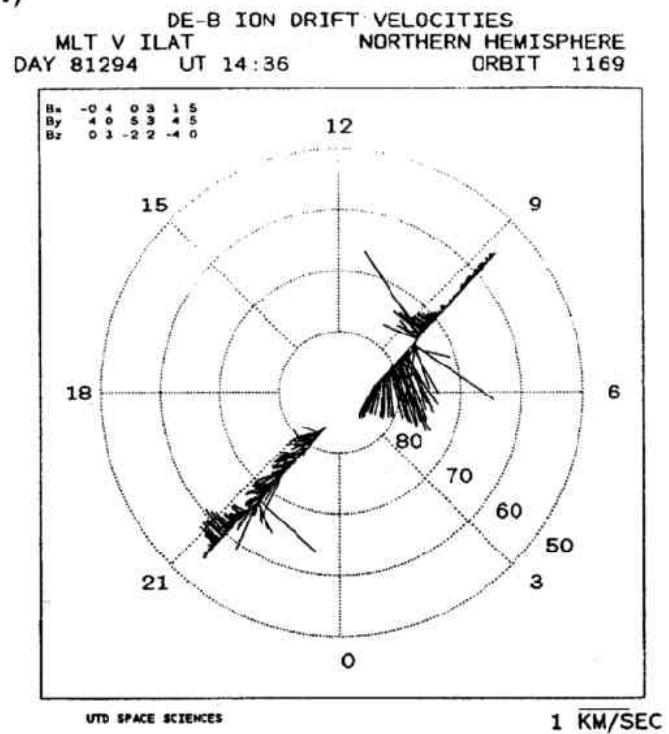

d.)

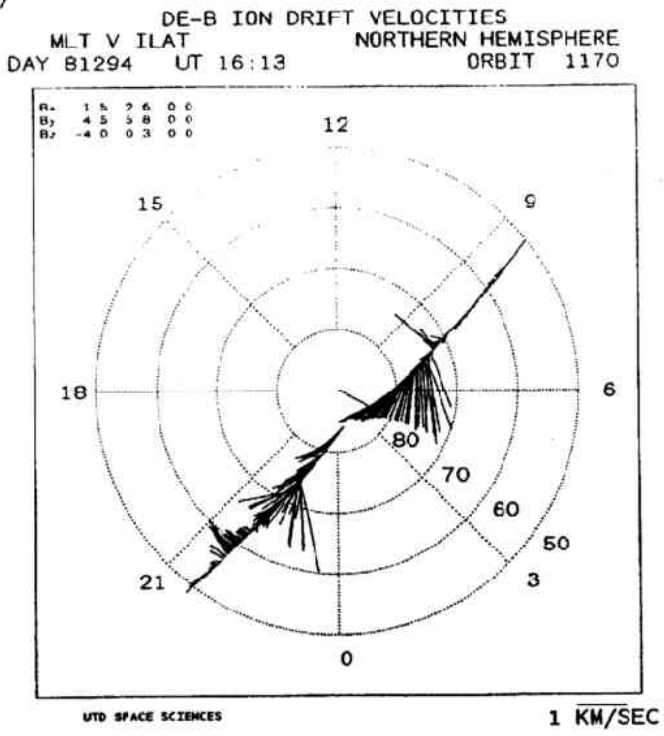

Fig. 3. Ion Drift data from the IDM/RPA for each of the 4 DE-2 passes. a) The growth phase pass. b) The pass occurring $\sim 10$ minutes after substorm onset. c) The early recovery phase pass near substorm maximum. d) The pass from later in the recovery phase.

\section{Results}

Figure 4 shows the resulting convection patterns from the ECPC model for each time the DE-2 spacecraft crossed the nightside polar cap boundary (center column), a corresponding sketch of the observed auroral oval from the DE-1 auroral imager (left column), and a cartoon sketch of the geomagnetic 
tail (right column). Table 1 lists the dayside and nightside gap potential drops for each pass. Also, listed is the orbit number of the DE-2 pass, the UT time the pass crosses the nightside polar cap boundary, the $A L$ index, the substorm phase, whether the polar cap is expanding or contracting, and the polar cap diameter. The reader should note that if the nightside gap potential drop is smaller (larger) than the dayside gap potential drop then the polar cap is expanding (contracting). A gap potential drop of zero indicates that the gap is closed and that dayside merging or tail reconnection has apparently ceased.

In the top row of Fig. 4, the growth phase convection appears as a two cell pattern with positive IMF $B_{y}$ asymmetry. Equipotentials are solid lines. The jagged line in the model marks the location of the polar cap boundary. The gaps are visible as breaks in this line. In the 13:39 convection pattern, the dayside gap

$13: 45$

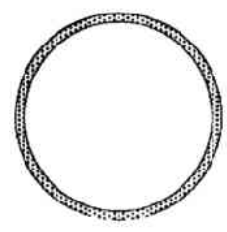

$14: 46$

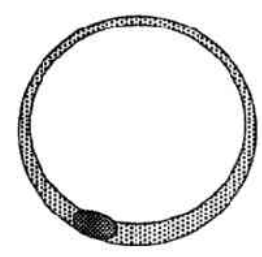

$15: 10$

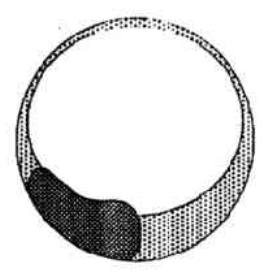

$16: 14$

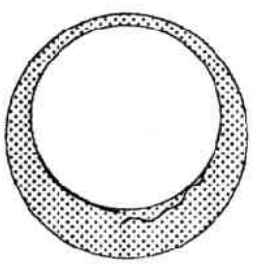

$13: 39$

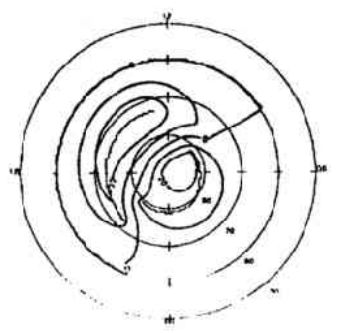

Growth Phase

$14: 42$

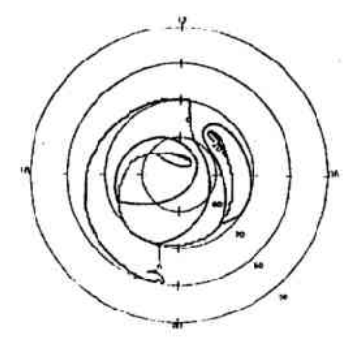

$15: 20$

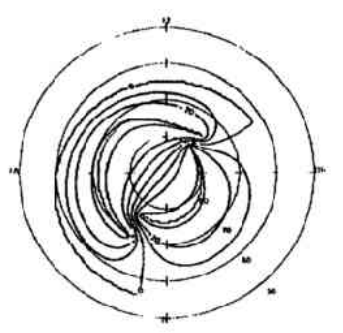

16:17

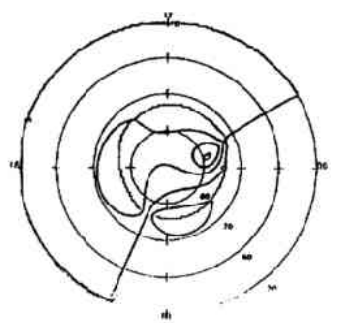

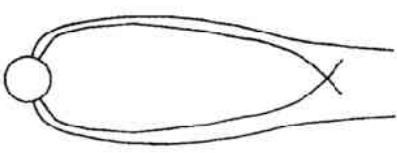

Just after onset

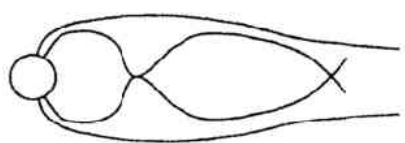

Near maximum

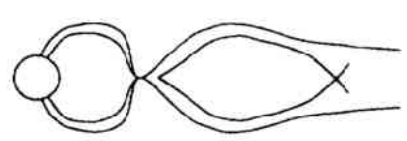

Recovery

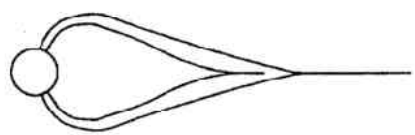

Fig. 4. Sketches of the auroral oval obtained from DE-1 images (left column), the resulting convection pattern snapshot as calculated with the ECPC model (center column), and a corresponding cartoon of the geomagnetic tail (right column). Dark areas represent the brightest portions of the auroral oval. The convection snapshots are presented on a polar dial. Solid lines show equipotentials. Jagged lines mark the location of the polar cap boundary. Times listed with the auroral sketches match the time of the DE-1 auroral image. The time listed next to the convection pattern is the time the DE-2 spacecraft crosses the nightside polar cap boundary for each pass modelled. 
Table 1. For the four passes in question on Oct. 21, 1981, we list the orbit number, the UT time the spacecraft crosses the poleward boundary of the nightside auroral oval, the $A L$ value at that time, the substorm phase, whether a nightside gap was required to model the data, whether the polar cap is determined to be expanding or contracting, the potential drops across both the nightside and dayside gaps, and the polar cap diameter determined by the LAPI particle data in degrees latitude.

\begin{tabular}{|c|c|c|c|c|c|c|c|c|}
\hline Orbit & UT & $A L(\mathrm{nT})$ & $\begin{array}{l}\text { Substorm } \\
\text { phase }\end{array}$ & N-gap? & Ex or contr.? & $\begin{array}{c}\mathrm{N} \text {-gap } \\
\text { potential drop }\end{array}$ & $\begin{array}{c}\text { D-gap } \\
\text { potential drop }\end{array}$ & $\begin{array}{l}\text { Polar cap } \\
\text { diameter }\end{array}$ \\
\hline $1168 \mathrm{~S}$ & $13: 39$ & $<-50$ & growth & $\mathrm{Y}$ & Ex. & 30 & 60 & 25 \\
\hline $1169 \mathrm{~N}$ & $14: 42$ & -250 & onset & $\mathrm{N}$ & Ex. & 0 & 50 & 35 \\
\hline $1169 \mathrm{~S}$ & $15: 20$ & -500 & recovery & $\mathrm{Y}$ & Contr. & 100 & 80 & 36 \\
\hline $1170 \mathrm{~N}$ & $16: 17$ & -250 & recovery & $\mathrm{Y}$ & Contr. & 40 & 0 & 34 \\
\hline
\end{tabular}

is broad covering 3 hours of local time in the morning sector. Concurrent to the convection, the observed auroral oval was uniform and rather dim. The polar cap is expanding as expected during the classical growth phase (McPherron, 1979) (Table 1). What is of particular interest about this pass is that the nightside gap is observed. This could indicate that tail reconnection is occurring at a distant neutral line before substorm onset as sketched in the third column of Fig. 4.

The second pass occurs 8 minutes after the substorm onset as shown in Fig. 4 , row 2 . The initial auroral brightening is observed at 14:34 UT. By 14:46 UT, the bright auroral patch has spread somewhat (left column). Again, there is a two-cell asymmetric convection pattern due to positive IMF $B_{y}$. The nightside gap configuration has vanished after the onset as supported by an observed decrease in the nightside electric fields. The polar cap is still expanding. As sketched in the third column of Fig. 4, the lack of open flux reconnection early in the expansion phase is attributed to the reconnection of closed flux with closed flux at the near-earth neutral line (Hones, 1984). Moses et al. (1989) in their statistical study of ionospheric convection during substorms noted that the nightside gap was only present $29 \%$ of the time during the expansion phase. This result could indicate that the nightside gap forms some time after the onset consistent with our result here.

In the third row of Fig. 4, the 15:20 UT DE-2 pass is featured and occurs during the early recovery phase. By this time, a strong nightside gap has formed (center column). Also in the auroral image in the left column, a large spiral structure has formed in the premidnight auroral oval. The DE-1 image was cut off duskward of a line from $2100-1200$ MLT. The full extent of the brightest portion of the auroral oval is therefore only speculation. From Table 1, the nightside gap potential drop is exceeding the dayside gap potential drop. Both potential drops are strong with the nightside gap potential drop exceeding the dayside gap potential drop. Thus, the polar cap is strongly contracting consistent with a net decrease of open lobe flux. In the tail (right column), open flux would be reconnecting with open flux.

In the 16:17 UT pass pictured in the fourth row of Fig. 4, the polar cap is also contracting. The dayside gap has closed and a lobe cell has formed near the dawn polar cap boundary (center column). The nightside gap has weakened in this later portion of the recovery phase. The auroral oval is still latitudinally broad but, quite dim with a few narrow arcs at the poleward edge (left column). The reconnection line may have retreated down the tail as in Fig. 4 in the right hand column.

\section{Discussion}

Overall, this particular isolated substorm has many of the characteristics of the classic substorm (McPherron, 1979). During the growth phase, the polar cap expands as open flux is added to the lobes. Early in the expansion phase, the nightside gap has not formed yet because the reconnection at the nearEarth neutral line only involves the distended closed flux which forms the plasmoid (Hones, 1984). During early recovery phase, the potential across the nightside gap and thus the rate of tail reconnection is still very strong. Later on in the recovery phase, the rate of tail reconnection slows. 
A superposed epoch analysis of the polar cap motion for 64 substorms was performed by Weimer $e t$ al. (1992). They found that the polar cap increases in radius during the growth phase by 6 degrees latitude on average. For one hour following substorm onset, the polar cap contracts returning to the pre-growth size.

In contrast to Weimer et al.'s (1992) average result, the polar cap does not contract much after substorm onset in the particular event studied here. Table 1 lists the diameter of the polar cap obtained from the LAPI data for each of the 4 passes. The polar cap grows considerably over the growth phase. But, the polar cap doesn't appear to return to the early growth phase diameter near the end of the event. Assuming a circular polar cap, this means a there was $\sim 50 \%$ increase in lobe flux from the first DE-2 pass to the last. At the end of the substorm, there is nearly as much energy stored in the lobes as at the onset. Because dayside merging doesn't turn off over the course of the event and the polar cap doesn't change size much after the onset of the substorm, dayside merging keeps pace with tail reconnection.

We can estimate the amount of flux reconnected on the nightside and merged on the dayside during the substorm by assuming the rate of flux crossing the gap remains steady from one satellite pass to the next. Equation (2) describes the rate at

$$
\Phi_{\text {gap }}=\int B_{\mathrm{i}} v_{\perp} d l
$$

which flux crosses the gap where $B_{\mathrm{i}}$ is the value of the ionospheric field, $v_{\perp}$ is the flow speed across the gap, and $\int d l$ is the length of the gap. Since $v_{\perp}$ is proportional to the potential drop across the gap, the rate at which flux crosses the gap is the gap potential drop.

In this case, we assume the nightside gap potential drop of $100 \mathrm{kV}$ is sustained for 1 hour (early recovery phase) and $40 \mathrm{kV}$ sustained for another $1 / 2$ hour (late recovery phase). Over the recovery phase, a maximum of $\sim 4 \times 10^{8}$ webers are removed from the lobes. Assuming the nightside gap potential drop remains steady until the next satellite pass gives us only a rough estimate of the reconnection rate since we expect the gap potential drop to evolve with time. Doing a similar calculation for the dayside merging rate, we obtain $\sim 4 \times 10^{8}$ webers of open flux added over expansion and recovery phase. This is in good agreement with the measured values of the polar cap diameter from LAPI which show little change in polar cap diameter during the recovery phase. Assuming a circular polar cap and that we cross through the center, the polar cap contained $\sim 7.4 \times 10^{8}$ webers when the polar cap is observed to be largest in the early recovery phase DE-2 pass. If no flux had been added to the polar cap in the recovery phase, the polar cap would have shrunk $>50 \%$. Despite the amount of open flux added by dayside merging, substantial energy is released during the substorm and in this case, particularly during the recovery phase.

Over the expansion and recovery phase, the polar cap size may not have changed much but it's location does. It should be noted that during the expansion and recovery phase of this event the nightside polar cap boundary moves $\sim 5^{\circ}$ poleward while the dayside polar cap boundary moves a similar amount equatorward. This gives the net effect of a sunward translation of the polar cap. Flux seems primarily added to the dayside portion of the polar cap while it is removed from the nightside.

A consistent theme to all studies using the ECPC model is that the nightside reconnection rate is strongest during the early recovery phase (i.e. the nightside gap potential drop is largest in early recovery). In the first Moses et al. (1989) statistical study with 39 events, the nightside gap was observed in only $29 \%$ of the expansion phase cases and $75 \%$ of the recovery phase cases leading them to conclude that the nightside gap probably forms after the onset of the substorm. Moses et al. (1994) observed that in a number of consecutive substorms that the magnitude of the nightside gap potential drop varied directly with the $A L$ index during the recovery phase. This result indicates that tail reconnection would be strongest during the maximum of the substorm and would continue but weaken through the recovery phase.

In this study of an isolated substorm, the nightside gap was not present 8 minutes after the onset of the substorm, i.e. the observed nightside electric field decreased after onset. Then, 40 minutes later during the recovery phase, the nightside gap was very strong. This observation suggests that the nightside 
gap closed near the onset time and reformed well into the expansion phase for this event. Early on in the substorm, closed flux may be reconnecting with closed flux to form a plasmoid.

Other authors have obtained similar results. Lockwood and Cowley (1992) noted that open flux removal from the polar cap was delayed 20-30 minutes after substorm onset in the CDAW-6 event. Also, Gazey et al. (1995) have reported the beginning of open flux reconnection 10 minutes after onset in coordinated observations from radar and ground magnetometers. Thus, the formation of the nightside gap may well be delayed after substorm onset.

\section{Conclusions}

In the event studied here, the beginning of open flux reconnection doesn't begin until $>8$ minutes after substorm onset. We observe a decrease in the nightside electric fields from the growth phase to the expansion phase. Nightside electric fields increase as the exchange of open flux reaches its observed maximum 40 minutes after onset in the recovery phase. It is apparent that even though the polar cap shrank very little during the recovery phase, there was substantial reconnection in the magnetotail. $\sim 4 \times 10^{8}$ webers were reconnected during the recovery phase of the substorm. This was $>50 \%$ of the polar cap flux when the polar cap was at it's largest extent.

The authors thank Dr. C. J. Owen for helpful comments. Research at Queen Mary and Westfield College was supported by PPARC (UK) grant GR/J88388. The work at UTD was supported by US Air Force Contract F1962893-K-0008.

\section{REFERENCES}

Craven, J. D. and L. A. Frank, The temporal evolution of a small auroral substorm as viewed from high altitudes with Dynamics Explorer 1, Geophys. Res. Lett., 12, 465-468, 1985.

Gazey, N. G. J., M. Lockwood, P. N. Smith, S. Coles, R. J. Bunting, M. Lester, A. D. Aylward, T. K. Yeoman, and H. Lühr, Development of substorm cross-tail current disruption as seen from the ground, J. Geophys. Res., 100, 9633-9648, 1995.

Hones, E. W., Plasma sheet behavior during substorms, in Magnetic Reconnection in Space and Laboratory Plasmas, pp. 178$184,1984$.

Lockwood, M. and S. W. H. Cowley, Ionospheric convection and the substorm cycle, in Substorms 1: Proceedings of the 1st International Conference on Substorms, ICS-1, Eur. Space Agency Special Publ. 335, pp. 99-109, 1992.

Lockwood, M., S. W. H. Cowley, H. Todd, D. M. Willis, and C. R. Clauer, Ion flows and heating at a contracting polar cap boundary, Planet. Space Sci., 36, 1229, 1988.

McPherron, R. L., Magnetospheric substorms, Rev. Geophys. Space Phys., 17, 657-681, 1979.

Moses, J. J. and G. L. Siscoe, Open flux merging in an expanding polar cap model, in Modelling Magnetospheric Plasma, edited by T. E. Moore and J. H. Waite, pp. 247-250, AGU Geophysical Monograph Scrics, 44, Washington, D.C., 1988.

Moses, J. J., G. L. Siscoe, N. U. Crooker, and D. J. Gorney, IMF $B_{y}$ and day-night conductivity effects in the expanding polar cap model, J. Geophys. Res., 92, 1193-1198, 1987.

Moses, J. J., G. L. Siscoe, R. A. Heelis, and J. D. Winningham, A model for multiple throat structures in the polar cap flow entry region, J. Geophys. Res., 93, 9785-9790, 1988.

Moses, J. J., G. L. Siscoe, R. A. Heelis, and J. D. Winningham, Polar cap deflation during a magnetospheric substorm, J. Geophys. Res., 94, 3785-3789, 1989.

Moses, J. J., J. A. Slavin, T. L. Aggson, R. A. Heelis, and J. D. Winningham, Modelling ionospheric convection during a major geomagnetic storm on Oct. 22-23, 1981, J. Geophys. Res., 99, 11017-11025, 1994.

Weimer, D. R., J. R. Kan, and S.-I. Akasofu, Variations of the polar cap potential as measured during magnetospheric substorms, J. Geophys. Res., 97, 3945-3951, 1992. 